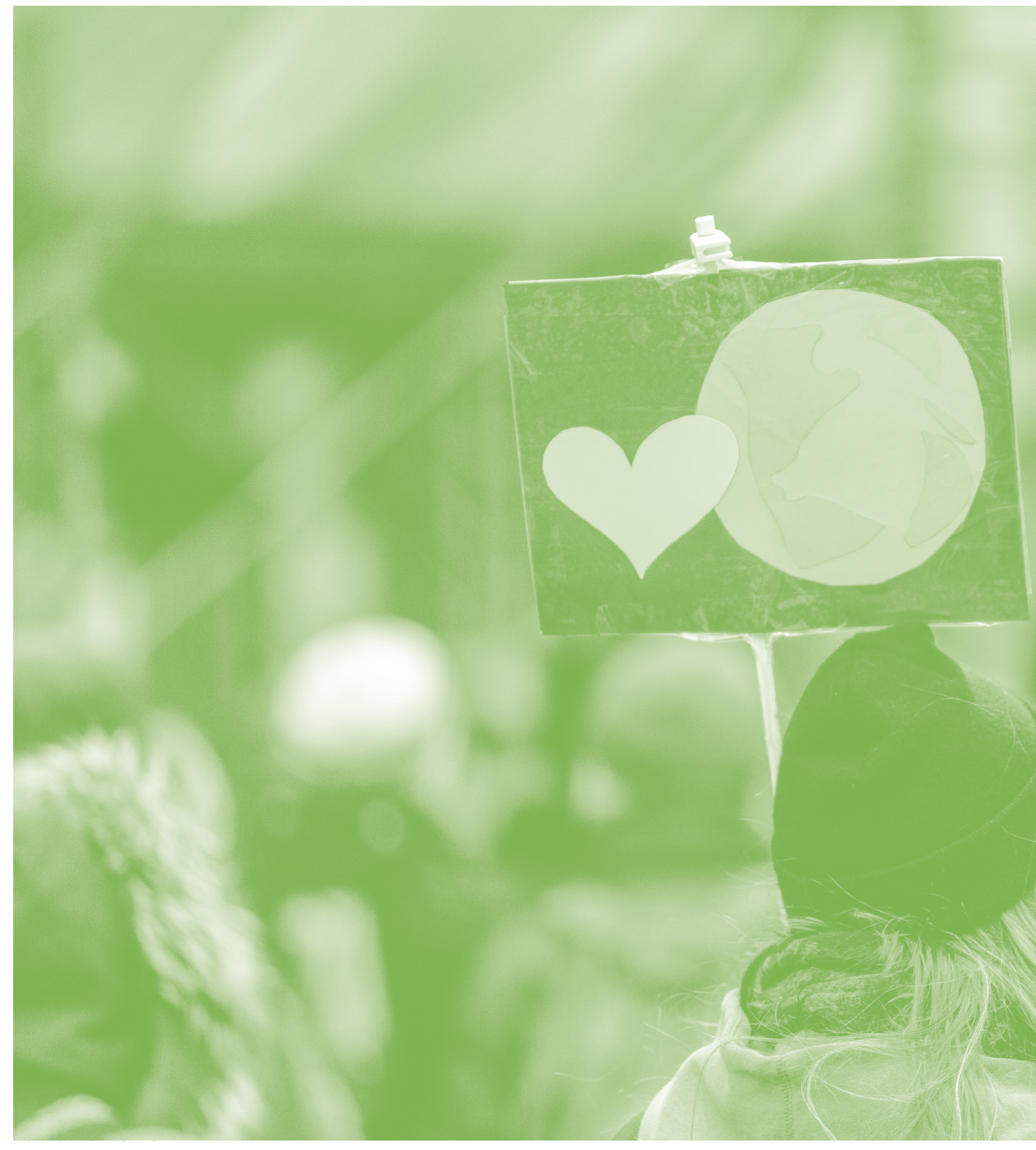

\title{
$\cdot 188$
}



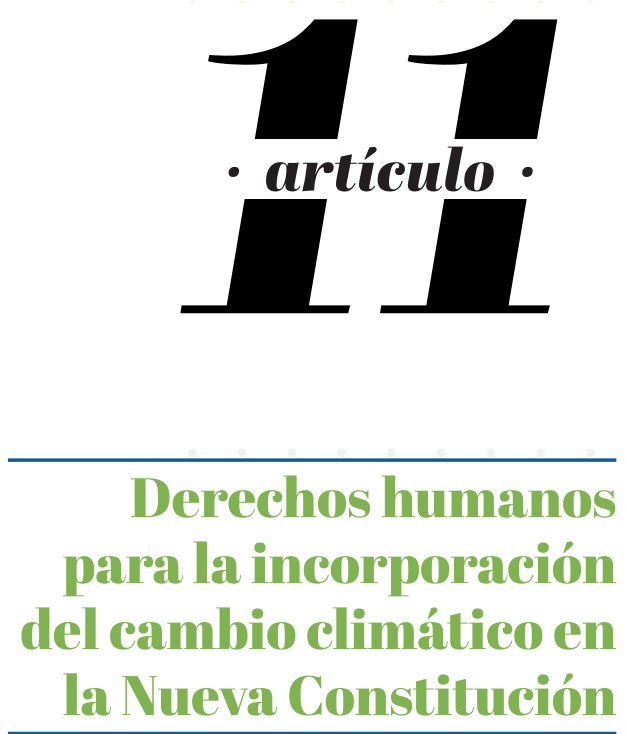

Pedro Cisterna ${ }^{464}$ y Ezio Costa ${ }^{465}$

DOI: 10.53110/WXIW8714

A. Introducción: La crisis climática y ecológica constituye el desafío principal de nuestra era, ${ }^{466}$ por lo que es necesaria su consideración y ponderación en cualquier transformación social, política y jurídica. El pueblo chileno se encuentra en un momento político e institucional histórico al discutir y definir una Nueva Constitución. Será la primera en nuestra historia nacida en democracia, la primera vez donde ciudadanos elegidos democráticamente tengan el mandato específico de escribir una Nueva Constitución, y la primera donde hombres y mujeres serán representados igualitariamente. Sin embargo, además de

464 Abogado de la Universidad de Concepción. LLM Global Environment and Climate Change Law, Universidad de Edimburgo. Actualmente, Candidato a PhD in Law en la Universidad de Edimburgo y asistente de investigación en la Escuela de Derecho de la Universidad de Edimburgo.

465 Abogado de la Universidad de Chile. Msc Regulation, London School of Economics. Doctor en Derecho de la Universidad de Chile. Profesor de Derecho, Universidad de Chile. Director ejecutivo Fiscalía del Medio Ambiente.

${ }^{466}$ Organización de las Naciones Unidas (ONU), 2018. 
todos estos sobresalientes elementos, la Nueva Constitución chilena será la primera que se escriba bajo un escenario en que la crisis climática es reconocida, convirtiéndose en una condicionante del proceso. Las instituciones que se creen con la Nueva Constitución deberían ser pensadas para hacer frente a la crisis climática, protegiendo nuestra comunidad y nuestros derechos.

El presente artículo toma la crisis climática y ecológica como un punto de partida, pero se centra particularmente en el fenómeno del cambio climático, analizando cómo este factor podría ser incluido efectivamente en la discusión constitucional. Los impactos ecológicos, sociales y jurídicos que el cambio climático tendrá sobre la sociedad chilena son reconocidos por nuestro Estado, así su consideración resulta esencial para definir los derechos e instituciones que sostendrán a la sociedad chilena durante las próximas décadas.

Bajo este marco, este trabajo argumentará, la importancia del reconocimiento de los derechos sociales para enfrentar los efectos del cambio climático. Primero, se explicará la relación que existe entre derechos humanos y cambio climático, y de este último con las obligaciones de derechos humanos de los Estados. En seguida, el artículo evidencia el impacto que el cambio climático tiene sobre los derechos humanos de poblaciones vulnerables. Finalmente, se propone la incorporación y desarrollo de un principio de acción climática como parte integrante de la Constitución Ecológica, y que incluye tres desafíos principales que la discusión constitucional debiese considerar. Estos desafíos son adaptación, mitigación y transición justa. Cada uno de estos desafíos es analizado, de cara a una transición socio-ecológica que se encuentre en línea con la protección de los derechos humanos. La propuesta de crear y consagrar un principio de acción climática obedece a la función que este tipo de normas cumple en la legislación, como orientadores de los objetivos que persiguen otras normas jurídicas y herramientas para la interpretación judicial. Un principio de este tipo sería consagrado por primera vez en una normativa constitucional y su construcción tiene que ver precisamente con las materias que se profundizan en el presente artículo.

B. Los Estados tienen obligaciones de derechos humanos consagradas en diversos tratados internacionales ${ }^{467}$ que definen cómo deben ser prote-

\section{Derechos hu- manos y cambio climático}


gidos, garantizados y promovidos, poniendo especial énfasis en las comunidades más vulnerables. ${ }^{468}$ Dichas obligaciones se ven complejizadas en el escenario actual de crisis climática, puesto que los efectos del cambio climático impactan el goce de los derechos humanos de las personas, ${ }^{469}$ como ha sido reconocido y documentado, tanto por organismos de derechos humanos como por instituciones internacionales de cambio climático. ${ }^{470}$

En efecto, la primera resolución sobre cambio climático del Consejo de Derechos Humanos de la ONU, ${ }^{471}$ enfatiza la amenaza que el calentamiento global constituye para los derechos humanos de las personas. El mismo órgano, en una posterior resolución, aclaró que estos impactos repercutirían más intensamente sobre los derechos humanos de las poblaciones más vulnerables. ${ }^{472}$ En seguida, es posible también encontrar este reconocimiento en los instrumentos internacionales del régimen climático, así el Acuerdo de París señala que las acciones climáticas de los Estados deben desarrollarse respetando, promoviendo y considerando sus respectivas obligaciones en derechos humanos. ${ }^{473}$

\section{i. Efectos del cambio climático sobre los derechos humanos y obligaciones de los Estados}

En el contexto de crisis climática, la oficina del Alto Comisionado de Derechos Humanos de la ONU se ha referido específicamente a algunos derechos humanos especialmente afectados. ${ }^{474}$ Entre ellos, el derecho a la vida, ${ }^{475}$ el derecho a una vivienda adecuada, ${ }^{476}$ el derecho a la alimentación, ${ }^{477}$ el derecho al agua y saneamiento, ${ }^{478}$ el derecho a la salud ${ }^{479}$ y el derecho a la libre determinación. ${ }^{480}$ Consideremos el derecho a la alimentación como ejemplo. El Panel Intergubernamental del Cambio Climático (PICC) ha determinado que, como consecuencia del cambio climático, se

diciembre de 1966, entrada en vigor 3 de enero de 1976) (PIDESC).

${ }^{468}$ Ver Bell, 2013, p. 162.

469 Desde una perspectiva filosófica, ver Gardiner, 2013; Bell, 2011. Desde una perspectiva legal, ver Knox, 2016. Desde una perspectiva científica, ver Walker, 2008.

470 Ver, por ejemplo, Decisión 1/CP.16, Acuerdo de Cancún.

471 Consejo de Derecho Humanos ONU, 2008.

472 Ver, Consejo de Derechos Humanos ONU, 2009.

${ }^{473}$ Ver, Acuerdo de París, preámbulo.

${ }^{474}$ Ver, Ibíd.

475 PICP, art. 6.

476 PIDESC, art. 12.

477 PIDESC, art. 11.

478 Asamblea General de la ONU, 2010.

479 PIDESC, art. 12.

480 PICP, art. 1. 
prevé una disminución de la producción de cultivo en latitudes más bajas. ${ }^{481}$ Esto aumentaría el riesgo de hambre e inseguridad alimentaria. Se proyecta que 600 millones de personas enfrentarían una fuerte malnutrición, especialmente en el África subsahariana. ${ }^{482}$ De no tomar medidas, las interferencias al goce y ejercicio de derechos humanos derivadas de los impactos del cambio climático podrían resultar en potenciales violaciones de estos últimos.

Es importante, sin embargo, distinguir entre una interferencia y una violación de derechos humanos. ${ }^{483}$ Ciertamente, el cambio climático y sus efectos interfieren con el goce de los derechos humanos de las personas, ${ }^{484}$ tales como el derecho a la vida, derecho a la salud, derecho a una vivienda adecuada, entre otros. ${ }^{485}$ Luego, para que se constituya una violación de derechos humanos debe configurarse un incumplimiento de los Estados en sus obligaciones de protección de estos derechos, cuestión que requiere un análisis particular.

Para graficar lo anterior, pensemos en una comunidad abatida por una inundación, perdiendo varias familias su vivienda. En el caso, en principio existe una interferencia del derecho a una vivienda adecuada producida por los impactos del cambio climático. Sin embargo, si dichas viviendas se encontraban en una zona de riesgo de inundación y contaban con un permiso de construcción para ello, podría existir una violación de derechos humanos porque el Estado no previno la vulneración del derecho a una vivienda adecuada. El Estado en cuestión debió evitar la construcción de viviendas en zonas de inundación o bien, haber desarrollado obras para mitigar cualquier amenaza climática. Por ende, en esta situación sí podría existir una violación de derechos humanos derivada de la omisión del Estado de prevenir que el derecho a una vivienda adecuada se protegiera.

Considerando entonces que, en una gran mayoría de los casos nos encontraremos frente a interferencias a derechos humanos producidas por los efectos del cambio climático, cabe preguntarse a qué estaría obligado un Estado ante dichas circunstancias y cuándo podríamos encontrarnos frente a un incumplimiento. Al respecto, las obligaciones en materias de derechos humanos y cambio climático nacen del deber de los Estados de tomar acciones para proteger a su población de los efectos del clima, distinguiéndose entre obligaciones sustantivas y procedimentales.

Las obligaciones sustantivas de los Estados se enfocan en la adopción de

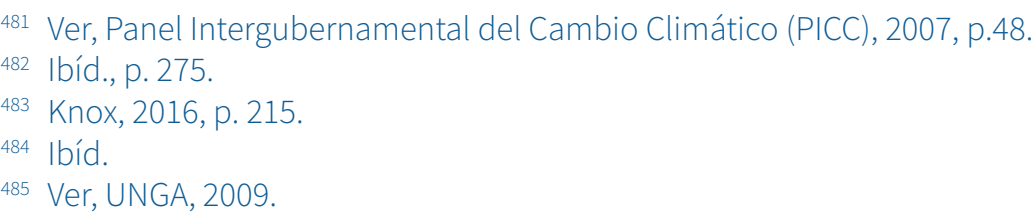


marcos legales e institucionales que respondan a las interferencias de los derechos humanos producidas por los efectos del cambio climático. ${ }^{486}$ En concreto, éstas se relacionan principalmente con la implementación de medidas de adaptación ${ }^{487}$ que protejan a la población de los efectos del cambio climático. Sobre esto, el Acuerdo de París establece en su artículo 7 que los Estados deben tomar acciones de adaptación tomando en consideración sus propias circunstancias. ${ }^{488}$

Luego, las obligaciones procedimentales ${ }^{489}$ se enfocan en los procesos necesarios para implementar medidas sustantivas, abarcando distintos focos. La primera sería la de evaluar los diferentes impactos que el cambio climático pueda generar, brindando un acceso público a dicha información. ${ }^{490}$ En segundo lugar, facilitar la participación de las comunidades en los procesos de decisión de materia ambiental y climática, con una especial consideración en los grupos marginalizados. ${ }^{491}$ Por último, entregar a las comunidades acciones legales que puedan aplicarse a casos de violación a derechos humanos derivadas de los efectos del cambio climático. ${ }^{492}$

Como es posible observar, las obligaciones procedimentales que se contemplan están alineadas con los derechos de acceso (a la información, la participación y la justicia en materia ambiental), los que a su vez son parte fundamental del derecho a vivir en un ambiente sano y especialmente relevantes en relación con los grupos vulnerables.

\section{ii. Derechos humanos y poblaciones vulnerables frente al cambio} climático

El régimen internacional de los derechos humanos ha establecido como prioridad especial la protección de poblaciones más vulnerables. ${ }^{493}$ Los grupos vulnerables se caracterizan por estar bajo situaciones de múltiple e invisibilizada discriminación, siendo necesarios instrumentos especiales para la protección de sus derechos humanos. Una vulnerabilidad principal que estos grupos enfrentan es la pobreza, expresada a través de diferentes carencias tales como la falta de alimentación adecuada, la baja calidad de educación o habitar en zonas ambientalmente degradadas. ${ }^{494}$ Desde el

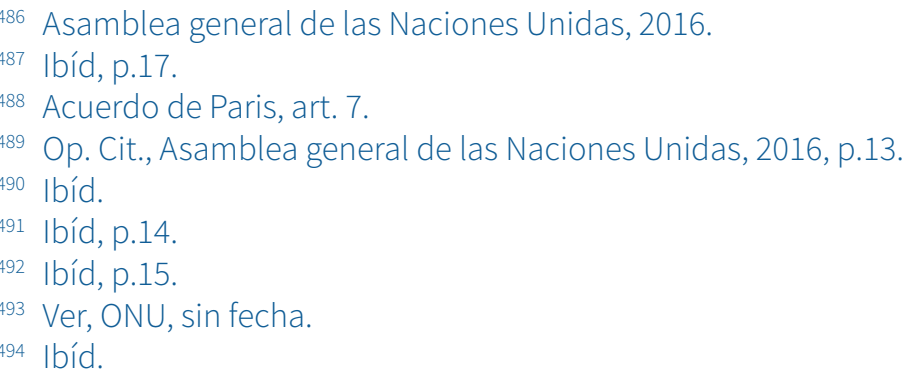


punto de vista ambiental, se ha observado que aquellos segmentos sociales que ya se encuentran viviendo en pobreza, verán agravada su situación por los efectos del cambio climático. ${ }^{495}$

El régimen internacional del cambio climático ha reconocido desde un inicio la situación de vulnerabilidad de algunas naciones y comunidades frente a los efectos del cambio climático. ${ }^{496}$ En este sentido, la Convención Marco de las Naciones Unidas para el Cambio Climático se refiere expresamente en su artículo 3.2 a la situación de especial vulnerabilidad que viven los países en desarrollo. ${ }^{497}$ En la misma línea, el PICC afirmó que las personas que son marginalizadas a nivel social, económico, político o institucional son especialmente vulnerables al cambio climático. ${ }^{498}$

Una clara definición de vulnerabilidad la entrega el Programa de las Naciones Unidas para el Desarrollo (PNUD) en su Informe "Solidaridad humana frente a un mundo dividido". ${ }^{999}$ El informe distingue entre riesgo y vulnerabilidad. El primero se refiere a la exposición a peligros externos sobre los cuales la población tenga control limitado. ${ }^{500}$ La vulnerabilidad en cambio, sería una medida de capacidad para controlar peligros sin sufrir pérdidas potencialmente irreversibles de bienestar. ${ }^{501}$ Bajo esta distinción es posible aseverar que el cambio climático constituye un riesgo global que implica una amenaza mayor para quienes son más vulnerables, es decir aquellos que tienen una menor capacidad para controlar peligros.

Luego, los factores de vulnerabilidad definidos por el PICC y los conceptos de riesgo y vulnerabilidad proporcionados por el PNUD se pueden ilustrar observando el Plan de Adaptación de Ciudades del Ministerio del Medio Ambiente de Chile. ${ }^{502}$ De acuerdo a dicho documento, la temperatura de Santiago hacia el 2050 aumentaría en un 14\% promedio en época estival. ${ }^{503} \mathrm{El}$ aumento promedio de temperatura en zonas urbanas se expresa en una mayor frecuencia de olas de calor ${ }^{504}$ y la población de Santiago estará expuesta al riesgo generado por estos incrementos de temperatura y las islas de calor urbano. ${ }^{505}$ Sin embargo, la vulnerabilidad de la población a

\footnotetext{
95 Asamblea General de las Naciones Unidas, 2009, pár. 67.

${ }^{96}$ Convención Marco de las Naciones Unidas sobre Cambio Climático(CMNUCC), 1992.

497 Ibíd., art. 3.2.

498 Panel Intergubernamental del Cambio Climático, 2014, p. 6.

99 Programa de las Naciones Unidas para el Desarrollo, 2007.

Ibíd, p. 71.

1 Ibíd.

Ministerio del Medio Ambiente, 2018.

so3 Ibíd., p. 23.

504 Para entender fenómeno de islas de calor urbana, ver, Kovats y Akhtar, 2009, p.166.

505 Ver Mohajerani, Bakaric y Jeffrey-Bailey, 2017, p. 522.
} 
estos fenómenos será distinta. El PICC ha señalado que las áreas verdes son útiles y necesarias para reducir los efectos de las "islas de calor urbano". ${ }^{506}$ En el caso de Santiago, la densidad de áreas verdes es notablemente mayor en las comunas más acomodadas, en contraste con las comunas más pobres, ${ }^{507}$ generando una mayor vulnerabilidad frente a los efectos de olas de calor a los sectores socialmente excluidos.

Los desafíos antes explicitados exigen considerar diversas dimensiones, siendo una de ellas la construcción de una estructura jurídica capaz de enfrentar el desafío climático, promoviendo el respeto y ejercicio de los derechos humanos. Dicha estructura debiera erigirse sobre lo que se ha conceptualizado como Constitución Ecológica. Esto es, normas constitucionales que pongan la protección del medio ambiente como un elemento central de nuestra organización jurídico-política, armonizando las actividades de la sociedad con las de la naturaleza.

En ese marco de acción, proponemos la incorporación de un principio de acción climática, que contenga las ideas de adaptación, mitigación y transición justa, ${ }^{508}$ que esta sección desarrollará. Un principio de acción climática constituiría una novedad para los ordenamientos nacionales, puesto que no es un principio que se encuentre actualmente formulado, sino una nomenclatura, que a nuestro juicio, puede recoger las obligaciones y objetivos de mitigación, adaptación y transición justa. Proponemos este principio sin la intención de rigidizar su contenido, sino más bien destacando su carácter emergente y flexible, facilitando la transversalidad de los desafíos y obligaciones en torno al cambio climático.

\section{i. La importancia de la Adaptación}

La Convención Marco de las Naciones Unidas para el Cambio Climático declaró como objetivo principal "la estabilización de las concentraciones de gases de efecto invernadero". ${ }^{509}$ Este enfoque en mitigación fue confirmado con el Protocolo de Kioto, que exigía principalmente a países desarrollados reducir sustancialmente sus emisiones. ${ }^{510}$ Sin embargo, el avance del cambio climático y la aparición más frecuente de sus efectos equilibró las estrategias de mitigación y adaptación para enfrentarlo. Desde la COP
C.

La acción climá tica como principio de acción de una Constitución Ecológica

506 Op. Cit., Panel Intergubernamental del Cambio Climático, 2014, p. 573.

507 Ver Pauta, 2019.

508 Ver ONG FIMA, 2020.

509 Ver, CMNUCC art. 3.

510 Ver, Protocolo de Kioto, art. 3.1. 
$7^{511}$ en Marrakech, comienza a discutirse la implementación de modelos de adaptación en países y comunidades más vulnerables, a través de los Planes Nacionales de Adaptación. Pasos posteriores importantes se dieron en la COP $10^{512}$ (Buenos Aires), COP11 $1^{513}$ (Montreal) y COP12 ${ }^{514}$ (Nairobi), que finalizaron con el lanzamiento del "Programa de Trabajo sobre impactos, vulnerabilidad y adaptación al cambio climático". ${ }^{515}$

En la COP16 (Cancún) la Adaptación tuvo un protagonismo especial estableciéndose el "Marco de Adaptación de Cancún"516 que, entre otras cosas, llamó a las partes a planificar, priorizar e implementar acciones de adaptación evaluadas bajo criterios sociales, económicos y ambientales. Finalmente, para monitorear las medidas exigidas por el Marco, se estableció un Comité de Adaptación. ${ }^{517}$

Los anteriores avances influyeron positivamente en la redacción del Artículo $7^{\circ}$ del Acuerdo de París, ${ }^{518}$ que regula la adaptación. Esta disposición establece como objetivo global la mejora de las capacidades adaptativas, la resiliencia y la reducción de la vulnerabilidad frente al cambio climático. ${ }^{519}$ La implementación del concepto de adaptación en el Acuerdo de París considera diversas escalas, nacional, sub-nacional y local. ${ }^{520}$ Además, llama a poner especial énfasis en la protección de comunidades vulnerables, estableciendo medidas que tomen en cuenta su propio conocimiento local. ${ }^{521}$

El énfasis de la adaptación en la protección de las comunidades vulnerables está fuertemente ligado al principio de equidad del régimen del cambio climático. Este principio busca la promoción de la justicia tanto entre las generaciones presentes ${ }^{522}$ como entre éstas y las futuras. Además, actúa a nivel interestatal a través del principio de responsabilidades comunes pero diferenciadas. ${ }^{523}$ Este último principio vela porque los Estados con una mayor responsabilidad en la generación del calentamiento global y con mayores niveles de desarrollo, asuman una mayor responsabilidad en la implementación de acciones contra el cambio climático.

Decisión 1/CP.7, 2001.

Decisión 1/CP.10, 2004

Decisión 1/CP.11, 2005.

14 Decisión 1/CP.12, 2006.

Decisión 6/CP.12, 2006.

Decisión 1/CP.16, 2010.

Ibíd.

18 Decisión 1/CP.21, Articulo 7.

19 Ibíd., pár. 1.

20 Ibíd., pár. 2.

221 Ibíd., pár. 5.

522 Shelton, 2008, p. 642.

523 Ibíd. Ver también, Cisterna Gaete, 2020, p. 39. 
Las inequidades reflejadas a nivel internacional son replicadas a nivel doméstico, ${ }^{524}$ ilustrándose, por ejemplo, en el nivel de emisiones de distintos grupos sociales con relación a su ingreso y en la capacidad adaptativa que diferentes grupos socioeconómicos tienen para confrontar efectos del cambio climático. ${ }^{525}$ En este contexto de desigualdad a nivel doméstico, la implementación del principio de equidad, especialmente en lo que se refiere a la adaptación, resulta urgente y necesaria. Esto implica reforzar y mejorar las condiciones de las poblaciones vulnerables para confrontar los efectos del cambio climático, cuestión sustentada bajo diferentes aspectos. En primer lugar, porque a diferencia de la mitigación, la adaptación tiene más injerencia en contextos nacionales o locales. ${ }^{526} \mathrm{El}$ impacto directo de medidas de adaptación en comunidades vulnerables está dado por el carácter local de las mismas y el potencial involucramiento de gobiernos locales en su implementación. 527

Adicionalmente, las medidas de adaptación implican un avance en el desarrollo socioeconómico de las comunidades, reduciendo su vulnerabilidad. ${ }^{528}$ Luego, la adaptación al cambio climático y el desarrollo socioeconómico son elementos complementarios. ${ }^{529}$ Así, el progreso de las medidas de adaptación puede tener un efecto más tangible en la protección de las comunidades más vulnerables. Un tercer argumento que refuerza el rol de la adaptación como una implementación concreta del principio de equidad, ${ }^{530}$ es que la adaptación es más efectiva para proteger los derechos humanos de las comunidades vulnerables. ${ }^{531}$

Desde un punto de vista más procedimental, el diseño e implementación de las medidas de adaptación requiere esencialmente de la participación de las comunidades involucradas. Las medidas de adaptación se enfocan en necesidades particulares de las vulnerabilidades de ciertos territorios y grupos humanos. La mayoría de las veces, estos grupos han sido excluidos de las esferas de poder y decisión en diversas formas. La adaptación, para esos territorios, significa un cambio significativo en sus formas de vida, cuestión que, si pretende ser respetuosa de los derechos humanos, debería darse bajo procesos de participación efectiva y diseñados "desde abajo".

La presencia, guía y acción del Estado es esencial para que las medidas de adaptación sean efectivas. Sin embargo, la determinación de dichas me-

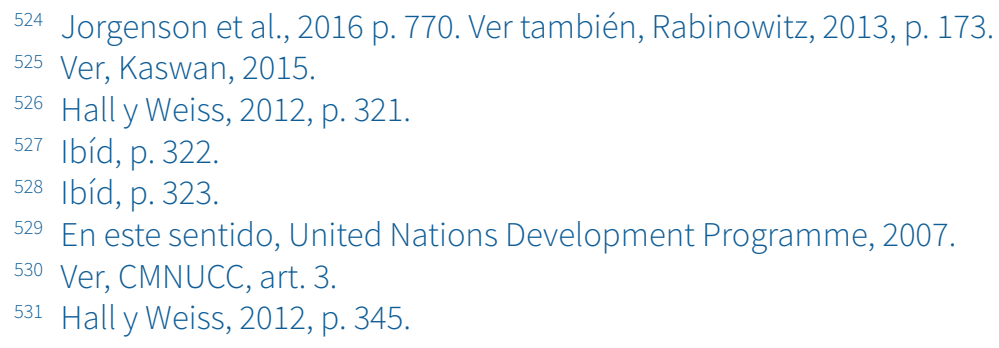


didas debe definirse principalmente por las personas y comunidades que habitan los territorios vulnerables. Así, no basta tan sólo con una inclusión simplemente informativa de estas comunidades, más bien se requiere una participación deliberativa, en coherencia con las exigencias del derecho ambiental y el Principio 10 de la Declaración de Río. ${ }^{532}$ En consecuencia, creemos que a través de la incorporación del principio de acción climática en la Nueva Constitución, se posiciona la adaptación como uno de los elementos centrales en la definición de políticas públicas y legislación. En específico, el reconocimiento del principio de acción climática puede permitir procesos de adaptación guiados por gobiernos locales (regionales, comunales o de cuencas), considerando una participación ciudadana efectiva junto a la información técnica correspondiente.

\section{ii. Mitigación}

La idea de la acción climática como un principio que podría encontrarse en nuestra constitución, impulsa tanto la adaptación como la mitigación, entendida esta última como la "intervención humana destinada a reducir las emisiones o mejorar los sumideros de gases de efecto invernadero". ${ }^{533}$

Este concepto, que en el leguaje climático parece tan específico, es a la vez un concepto que contiene una serie de acciones que contribuyen a la protección ambiental de un territorio. En efecto, la disminución de las actividades que emiten gases de efecto invernadero puede reducir las actividades contaminantes en general, mientras que la protección o recuperación de sumideros de carbono, puede implicar el cuidado de espacios naturales y de la biodiversidad en general. Lo anterior es una posibilidad y no una certeza, puesto que la manera en que el régimen climático define mitigación permite que los objetivos sean cumplidos por medios tecnológicos distintos de soluciones basadas en la naturaleza, siendo estas últimas las que creemos más esenciales de potenciar.

Las soluciones basadas en la naturaleza constituyen acciones climáticas y ambientales basadas en las propias funciones ecosistémicas, potenciando la protección y recuperación de los mismos. En general, se reconoce que también estas soluciones pueden ir acompañadas de acciones que no tengan el mismo carácter, constituyendo un enfoque integrado. ${ }^{534}$ Entre

Costa, 2020.

Panel Intergubernamental del Cambio Climático, 2018, p.85.

En esta misma línea avanzan las definiciones de la Unión Internacional para

la Conservación de la Naturaleza (IUCN): https://www.iucn.org/node/28778 y

el Banco linteramericano de Desarrollo (BID): https://publications.iadb.org/ publications/english/document/Nature-based_Solutions_Scaling_Private_Sector_Uptake_for_Climate_Resilient_Infrastructure_in_Latin_America_ 
las soluciones basadas en la naturaleza encontramos enfoques como la infraestructura verde, la reforestación, la conservación, el manejo sustentable y la gestión de cuencas.

Son este tipo de soluciones las que mejor dialogan con un cambio en la relación que tenemos con la naturaleza. Por lo tanto, en la medida que la Nueva Constitución propicie e incentive este cambio, probablemente las soluciones basadas en la naturaleza se conviertan en una de las herramientas esenciales para propiciar la mitigación.

La obligación de mitigar el cambio climático debiera comprender entonces, por una parte, la responsabilidad para con la humanidad, expresada en diversos tratados internacionales, de detener la emisión de gases de efecto invernadero, para desacelerar y finalmente frenar el fenómeno. Por otra parte, e igualmente fundamental, es la dimensión local de la mitigación, ya que las medidas de mitigación pueden convertirse en medidas de descontaminación o restauración de ecosistemas, atacando las actividades locales contaminantes.

\section{iii. Transición Justa}

El presupuesto de la justicia ambiental, es que las cargas y los beneficios ambientales puedan ser distribuidos de manera equitativa, evitando que existan personas privadas de los elementos básicos para la subsistencia, que son proveídos por la propia naturaleza. ${ }^{535} \mathrm{Ni}$ tampoco personas que carguen desproporcionadamente con los impactos ambientales que suponen algunas actividades productivas. ${ }^{536}$ Así, la transición justa es entendida como aquella noción que integra la equidad en los procesos asociados a lograr un mundo bajo en carbono, transformando las estructuras sociales y económicas que generan desigualdad. ${ }^{537}$

El anterior no ha sido un principio particularmente respetado, cuestión que en parte obedece a que su conceptualización es posterior a la existencia de una distribución de cargas y beneficios desigual. En materia climática, esto se evidencia en que las comunidades vulnerables son las que menos han contribuido a la generación del cambio climático y quienes han sufrido y sufrirán con mayor gravedad los efectos locales del cambio climático.

En un momento de transición a economías bajas en carbono, y esperablemente hacia un nuevo modelo económico, las variables distributivas de esta transición deben ser miradas con atención. Es esencial no repetir los

and_the_Caribbean.pdf.

535 Ver, MacLennan y Perch, 2012, p. 283.

536 Hervé, 2015, p. 38.

537 Pinker, 2020, p. 9. 
mismos errores del pasado y compensar a quienes han sufrido en sus territorios y en sus cuerpos los efectos de un modo de producción y consumo que no respetó los límites de la naturaleza. La lógica de la transición justa precisamente debería apuntar hacia la corrección de dichas problemáticas. Fijándose en no volver a cargar ni beneficiar a quienes ya estuvieron en esas posiciones respectivas, y al mismo tiempo, reforzar una restauración de ecosistemas y reparación de los daños producidos a las personas.

En seguida, existe una dimensión particular de la transición justa, relacionada con los y las trabajadoras de industrias que ya no resultan viables a la luz de la crisis climática y ecológica. Esta fuerza laboral ha sido el motor del bienestar material para ciertos grupos humanos y territorios. Esta dimensión es la más presente en las negociaciones y acuerdos internacionales sobre cambio climático. ${ }^{538}$ Por ejemplo, en Marzo de 2020, Chile suscribió la actualización de sus contribuciones nacionales determinadas (NDC) a la Secretaría de la Convención Marco de las Naciones Unidas para el Cambio Climático y uno de sus pilares esenciales e innovadores fue la incorporación de la justicia transicional en esta variable. ${ }^{539}$ Este concepto exige anticiparse a las consecuencias sociales que derivan de la transformación hacia economías bajas en carbono. El Acuerdo de París menciona este concepto en su preámbulo, reforzando la idea de "una reconversión justa de la fuerza laboral y de la creación de trabajo decente". ${ }^{540}$

La transición hacia una economía verde en Chile implicará pérdida de trabajo o reemplazo de mano de obra en muchos sectores del país, afectando a familias dependientes de dichas industrias. Además, políticas verdes mal implementadas pueden generar efectos económicos negativos en sectores medios y vulnerables de nuestra sociedad. Un claro ejemplo de lo anterior es el movimiento francés de los chalecos amarillos, nacido a raíz de la no consideración de las consecuencias que un impuesto al combustible podía traer para sectores más vulnerables del país. El nivel de movilización social y desestabilización que trajo una política verde impuesta verticalmente y sin criterios de transición justa no sólo puso en jaque al gobierno de Macron, sino también la legitimidad de implementar políticas sostenibles en democracias modernas.

Así, el concepto de justicia transicional viene a contribuir en evitar o mitigar los efectos adversos de políticas climáticas. Considerar una transición justa hacia políticas climáticas es esencial para la construcción de ordenes democráticos con garantía de derechos como a los que aspira la Nueva Constitución chilena. El concepto de justicia transicional refleja cómo la

538 Ver, Acuerdo de París, preámbulo.

539 República de Chile, 2020, pp. 23 y siguientes.

540 Acuerdo de París, preámbulo 
crisis de las democracias, el medio ambiente y el respeto a los derechos humanos están conectados, y su solución también. ${ }^{541}$

A nivel comparado, un instrumento emergente en el diseño e implementación de justicia transicional son las denominadas "Comisiones de Transición Justa”. ${ }^{542}$ El origen histórico de estas instancias en procesos de justicia transicional está relacionado con dictaduras o regímenes represivos que violaron derechos humanos, como el Apartheid en Sudáfrica. Sin embargo, recientemente en Canadá (2018) y Escocia (2019) establecieron estas comisiones para enfrentar el desafío climático transicional. Destacable es el caso escocés donde no sólo está dentro de los objetivos el hacerse cargo de la fuerza laboral afectada, sino también utilizar estas comisiones para profundizar, en general, la acción climática del gobierno. ${ }^{543}$

Como parte fundamental de la acción climática, incluir el concepto de justicia transicional, en una visión amplia, empuja a que las políticas públicas climáticas tengan variables distributivas a la vista, abriendo las puertas al diseño de estrategias preventivas que garanticen dignidad y respeto a los derechos humanos de quienes se vean afectados por las transformaciones ecológicas de nuestras economías. Lo anterior, nos parece de la mayor importancia cuando observamos que el movimiento de transformación socio-ecológica actual ha estado más marcado por la compensación a quienes han generado la crisis climática, que a quienes sufren sus consecuencias, cuestión que parece inaceptable en una visión mínima de justicia. ${ }^{544}$

Hay un aspecto innegable de colectividad en todo esto. Si bien quienes han profitado a sabiendas de la destrucción de los ciclos y estructuras de la naturaleza tienen una mayor responsabilidad sobre los resultados de sus acciones, no es menos cierto que varias de esas actividades fueron activamente promovidas por parte de la comunidad organizada en el Estado, siendo entonces que hay también una responsabilidad colectiva en volver las cosas a su lugar y reparar lo dañado. La paz social futura requiere también de actos de reconocimiento y reparación.

\footnotetext{
541 Ghaleigh, 2020, p. 434

542 Ibíd., p. 449.

543 Ver, ibíd., pp. 449-450

544 Esto es posible de observar muy claramente en el plan de descarbonización de Chile, donde los acuerdos voluntarios entre el gobierno y las empresas generadoras de energía en base a carbón han estado en la línea de asegurarles algún tipo de compensación. Al mismo tiempo, siguen existiendo zonas de sacrificio ambiental y el peor de los casos es del de la bahía de Quintero, donde incluso existe un fallo de la Corte Suprema (rol 5.888-2019) que ordena acciones para la solución del problema, las que -en gran parte- no han sido tomadas por el Estado a más de 2 años de haberse ordenado.
} 
D. El texto se ha enfocado en potenciar pilares de acción climática y la esencialidad de su consideración en la discusión constitucional para promover el reconocimiento y ejercicio de derechos humanos. El contexto de crisis climática a nivel global, repercute directamente en los caminos que nuestra Nueva Constitución debe definir para hacerse cargo de los desafíos ecológicos y sociales que esta crisis conlleva. Así, creemos necesario utilizar los conceptos de adaptación, mitigación y transición justa como orientadores de los debates de la Convención Constituyente que garanticen el respeto y ejercicio de derechos humanos en un contexto de crisis climática.

Hemos agrupado estos tres conceptos en torno al propuesto principio de acción climática. La incorporación de este principio en las bases de la institucionalidad de una Nueva Constitución, constituiría un avance en relación con la organización jurídica e interpretación de otras normas, que facilite y dinamice las respuestas de nuestro Estado frente a la crisis climática.

La evidencia irrefutable de que las comunidades más vulnerables serán las más afectadas por los efectos del cambio climático y permanente continuidad de la actividad extractiva, nos obligan a asumir medidas de mitigación y adaptación urgentemente. Además, los costos sociales y culturales de caminar hacia economías verdes requieren políticas públicas de adecuada respuesta y basadas en una transición justa, evitando que un nuevo modelo de desarrollo verde no profundice las desigualdades que provocaron el estallido social.

La consideración del cambio climático es un factor esencial para el logro de una justicia social sobre la que se erija esta nueva Carta Fundamental. El cambio climático, como toda crisis, nos entrega la oportunidad de replantear y profundizar nuestra democracia. Así, la Nueva Constitución y el cambio climático nos otorgan una doble oportunidad. Primero, la oportunidad de re-establecer nuestras relaciones sociales, económicas y principalmente políticas. Segundo, desarrollar este re-establecimiento considerando la crisis climática como un factor esencial para proyectar un país que responda a las necesidades de su pueblo y garantice los derechos humanos de las personas en un contexto de cambio climático. 
Asamblea General de las Nacion (2010)

64/292. El derecho humano al agua y al saneamiento. Documento No. A/RES/64/292.

Asamblea General de las Naciones Unidas (2009)

El derecho a una vivienda adecuada. Documento No. A/64/255.

Asamblea General de las Naciones Unidas (2016)

Informe del Relator Especial sobre la cuestión de las obligaciones de derechos humanos relacionadas con el disfrute de un medio ambiente sin riesgos, limpio, saludable y sostenible. Documento No. A/ $\mathrm{HRC} / 31 / 52$.

Bell, D. (2011)

'Does Anthropogenic Climate Change Violate Human Rights?', Critical Review of International Social and Political Philosophy, 14(2):99-124.

Bell, D. (2013)

'Climate Change and Human Rights', Wiley Interdisciplinary Reviews, 4(3):159-170.

Cisterna Gaete, P. (2020)

'La esencialidad del principio de responsabilidades comunes pero diferenciadas en el régimen internacional del cambio climático', Revista de Derecho Ambiental, 13:39-58.

Consejo de Derechos Humanos (2008)

Resolución 7/23. Derechos humanos y cambio climático. Documento No. A/HRC/RES/7/23.

Consejo de Derechos Humanos (2009)

Resolución 10/4. Los derechos humanos y el cambio climático. Documento No. A/RES/10/4.

Convención Marco de las Naciones Unidas sobre Cambio Climático (1992).

Convención Marco sobre el Cambio Climático (2001)

1/CP.7. Declaración Ministerial de Marrakech. Documento No. FCCC/ CP/2001/13/Add.1. 
Convención Marco sobre el Cambio Climático (2004)

1/CP.10 Programa de trabajo de Buenos Aires sobre las medidas de adaptación y de respuesta. Documento No. FCCC/CP/2004/10/Add.1.

Convención Marco sobre el Cambio Climático (2005)

1/CP.11 Diálogo sobre la cooperación a largo plazo para hacer frente al cambio climático mediante una mejor aplicación de la Convención. Documento No. FCCC/CP/2005/5/Add.1.

Convención Marco sobre el Cambio Climático (2006)

1/CP.12 Nuevas orientaciones para la entidad encargada del funcionamiento del mecanismo financiero de la Convención, destinadas a la administración del Fondo especial para el cambio climático. Documento No. FCCC/CP/2006/5/Add.1.

Convención Marco sobre el Cambio Climático (2006)

6/CP.12 Continuación de las actividades conjuntas realizadas en la etapa experimental. Documento No. FCCC/CP/2006/5/Add.1.

Convención Marco sobre el Cambio Climático (2010)

1/CP.16 Acuerdos de Cancún: resultado de la labor del Grupo de Trabajo Especial sobre la cooperación a largo plazo en el marco de la Convención. Documento No. FCCC/CP/2010/7/Add.1.

Convención Marco sobre el Cambio Climático (2015)

1/CP.21 Aprobación del Acuerdo de París. Documento No. FCCC/ CP/2015/10/Add.1.

Costa (2020)

Participación Ciudadana. Conceptos Generales, Deliberación y Medio Ambiente. Santiago de Chile: Ediciones Der.

Gardiner, S. (2013)

'Human Rights in a Hostile Climate', en Holder, C. y Reidy, D. (eds.) Human Rights: The Hard Questions. Cambridge: Cambridge University Press.

Ghaleigh, N.S. (2020)

'Just Transitions for Workers: When Climate Change Met Labour Justice', en Bogg, A., Rowbottom, J. y Young, A.L. (eds.) The Constitution of Social Democracy: Essays in Honour of Keith Ewing. Oxford: Hart Publishing, pp. 429-452.

Gobierno de Chile (2020)

Contribución determinada a nivel nacional (NDC) de Chile.

Hall, M. y Weiss, D. (2012) 
'Avoiding Adaptation Apartheid: Climate Change Adaptation and Human Rights Law', Yale Journal of International Law, 37(309).

Hervé Espejo, D. (2015)

Justicia Ambiental y Recursos Naturales. Valparaíso: Ediciones Universidad de Valparaíso.

Jorgenson, A. et al. (2016)

'Domestic Inequality and Carbon Emissions in Comparative Perspective', Sociological Forum, 31(S1):770-786.

Kaswan, A. (2015)

'Adaptation Justice', Social Science Research Network. ID: 2703631.

Knox, J. (2016)

'Human Rights Principles and Climate Change', en Gray, K., Tarasofsky, R. y Carlarne, C., The Oxford Handbook of International Climate Change Law. Oxford: Oxford University Press.

Kovats, S. y Akhtar, R. (2009)

'Climate, Climate Change and Human Health in Asian Cities' en Bicknell, J., Dodman, D. y Satterthwaite, D., Adapting Cities to Climate Change: Understanding and Addressing the Development Challenges. Londres: Earthscan.

MacLennan, M. y Perch, L. (2012)

'Environmental Justice in Latin America and the Caribbean: Legal Empowerment of the Poor in the Context of Climate Change', Climate Law, 3(3-4): 283.

Ministerio del Medio Ambiente (2018)

Plan de Adaptación al Cambio Climático para Ciudades 2018-2022.

Disponible en: https://mma.gob.cl/wp-content/uploads/2018/06/

Plan-CC-para-Ciudades_aprobado-CMS-ene2018-1.pdf (Consulta: 22 agosto 2021).

Mohajerani, A., Bakaric, J. y Jeffrey-Bailey, T. (2017)

'The Urban Heat Island Effect, Its Causes, and Mitigation, with Reference to the Thermal Properties of Asphalt Concrete', Journal of Environmental Management, 197:522-538.

ONG FIMA (2020)

Bases para una constitución ecológica. Disponible en: https:// www.fima.cl/wordpress/wp-content/uploads/2020/11/BASES-PARA-UNA-CONSTITUCIO\%CC\%81N-ECOLO\%CC\%81GICA-v.-25.11.20-1.pdf (Consulta: 22 agosto 2021). 
Organización de Naciones Unidas (sin fecha)

Secretary-General's Remarks on Climate Change [as Delivered]. Disponible en: https://www.un.org/sg/en/content/sg/statement/2018-09-10/secretary-generals-remarks-climate-change-delivered (Consulta: 22 agosto 2021).

Organización de Naciones Unidas (sin fecha)

The Resource: Part V. Persons with Disabilities and Multiple Discrimination - Rights of Special Groups, 9/10. Disponible en: https://www. un.org/esa/socdev/enable/comp508.htm (Consulta: 22 agosto 2021).

Pacto Internacional de Derechos Civiles y Políticos (1966).

Pacto Internacional de derechos económicos, sociales y culturales (1966).

Panel Intergubernamental del Cambio Climático (2007) Fourth Assessment Report.

Panel Intergubernamental del Cambio Climático (2014) Fifth Assessment Report.

Panel Intergubernamental del Cambio Climático (2018) Calentamiento global de $1,5^{\circ} \mathrm{C}$

Pauta (2019)

'Cómo se mide la desigualdad de los parques en las ciudades de Chile', Pauta, 12 de septiembre. Disponible en: https://www.pauta.cl/ calidad-de-vida/estadisticas-parques-espacios-verdes-ciudades-regiones-de-chile (Consulta: 22 agosto 2021).

Pinker, A. (2020)

Just transitions. A Comparative perspective. Report prepared for the Just Transition Commission.

Programa de las Naciones Unidas para el Desarrollo (2007)

La lucha contra el cambio climático: Solidaridad frente a un mundo dividido.

Protocolo de Kioto de la Convención Marco de las Naciones Unidas para el Cambio Climático (1997).

Rabinowitz, D. (2013)

'In-Country Disparities in Greenhouse Gas Emissions and Their Significance for Politicizing a Future Global Climate Pact Reaching International Cooperation on Climate Change Mitigation', Theoretical Inquiries in Law, 14(1):173-189.

Shelton, D. (2008)

'Equity', en Bodansky, D. et al. (ed.), The Oxford Handbook of Interna- 
tional Environmental Law. Oxford: Oxford University Press.

UNGA (2009)

Report of the Office of the United Nations High Commissioner for Human Rights on the relationship between climate change and human rights. Documento No. A/HRC/10/61.

United Nations Development Programme (2007)

Human Development Report 2007-2008, Fighting climate change: Human Solidarity in a divided world.

Walker, G. y King, D. (2008)

The Hot Topic: How to Tackle Global Warming and Still Keep the Lights On. Bloomsbury Publishing. 